\title{
Fenomeni troposferici di trasporto e diffusione a grande scala posti in evidenza da traccianti radioattivi
}

\author{
Giorgio Fea - adriano Gazzola
}

Ricevuto il 15 Giugno 1961

\section{Premessa.}

In Meteorologia, tra $i$ problemi che presentano maggior difficoltà, sia per l'indagine teorica che per quella sperimentale, vi sono quelli relativi alla determinazione delle traiettorie delle masse d'aria nei loro moti orizzontali e verticali, e quelli relativi alla valutazione dei fenomeni di diffusione che accompagnano tali moti.

Si pensi che le traiettorie, relativamente brevi nei fenomeni "locali", giungono a compiere anche uno o più volte il giro del globo.

Analogamente, e in particolare, $i$ fenomeni di diffusione si svolgono a scale estrev: camente diverse: da quella submicroscopica molecolare degli strati aderenti al suolo, a quella continentale od emisferica delle onde cicloniche.

Infatti nei processi di "scambio" fra strati sovrapposti o adiacenti, da percorsi del milionesimo di millimetro e tempi di pereorso del centomilionesimo di secondo, si passa a percorsi di migliaia e decine di migliaia di chilometri con tempi dell'ordine del giorno o della settimana.

Cosicché $i$ relativi coefficienti di diffusione variano fra valori $i$ cui estremi stanno fra loro come uno a un migliaio di miliardi.

Tra $i$ fenomeni permanenti o saltuari, dovuti alla natura o agli esperimenti dell'uomo, che possono agevolure le ricerche sui moti delle masse d'aria e sulla loro diffusione, sono di particolare ausilio quelli della radioattività naturale e della radioattività artificiale.

Le sostanze radioattive possono infatti essere utilizzate nell'atmosfera come "traccianti", in modi analoghi a quelli coi quali esse sono utilizzate negli studi di biologia e medicina. 
Il lavoro che si presenta costituisce appunto un tentativo di indagine sommaria sulle possibilita di studio delle reali traiettorie seguite dalle masse d'aria che si trovavano sulla zona dell'esplosione nucleare francese avvenuta nell'interno del Sahara il 13 Febbraio del 1960. In quel momento infatti l'armosfera, particolarmente priva di prodotti nucleari artificiali, ben si prestava a porre in evidenza quantitativi anche piccolissimi di tali sostanze.

Già in occasione del X Convegno dell'Associazione Geofisica Italiana sono stati esposti i primi risultati degli studi in corso presso la Divisione Studi e Ricerche del Servizio Meteorologico dell'A.M. sulla circolazione nella troposfera, studi condotti avvalendosi come traccianti dei prodotti di fissione emessi nelle prime due esplosioni nucleari francesi.

Sull'argomento sono stati pubblicati due articoli: il primo sugli Atti del X Convegno dell'A.G.I. ( $\left.{ }^{1}\right)$; il secondo, più ampio, sulla Rivista di Meteor. Aeronaut. ( $\left.{ }^{2}\right)$.

Da parte sua I. Brauer, del Servizio Meteorologico Tedesco, ha condotto uno studio analogo al nostro sulle conseguenze della prima esplosione nucleare francese $\left(^{\circ}\right)$; tale lavoro è giunto a nostra conoscenza solo di recente. In esso sono state tratte conclusioni che in parte concordano con quelle da noi dedotte ed in parte contrastano con esse. In questa relazione discuteremo i punti di contatto e quelli controversi; è bene premettere che $\mathrm{i}$ dati su cui i due studi sono basati si integrano a vicenda, in quanto i tedeschi erano a conoscenza dei dati non posseduti da noi e viceversa.

Per facilitare la lettura di quanto seguirà si ritiene opportuno riepilogare brevemente le nostre opinioni, riportando dall'articolo precedentemente pubblicato sulla Rivista di Meteorologia Aeronautica alcune parti essenziali ed alcune figure.

L'esame dei dati di radioattività e quello delle situazioni meteorologiche esistenti nel periodo successivo all'esplosione ci ha indotto a distinguere i quattro casi di trasporto di polveri radioattive schematizzati in Fig. 1. Di questi quattro casi i primi tre sono i più importanti. Essi presentano le seguenti caratteristiche fondamentali:

Tipo A: Trasporto nell'alta troposfera per mezzo delle correnti occidentali ed in particolare nel getto. Il moto è generalmente veloce e la radioattività aumenta rapidamente con la quota.

Tipo B: Trasporto nella bassa e media troposfera, con convogliamento nelle grandi celle anticicloniche subtropicali (alisei). Il moto è rela- 

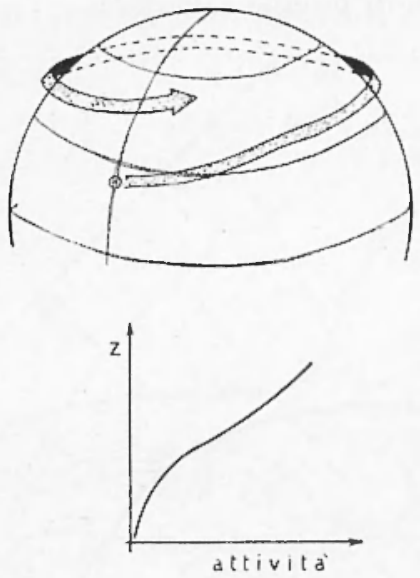

$S A B B \mid A$

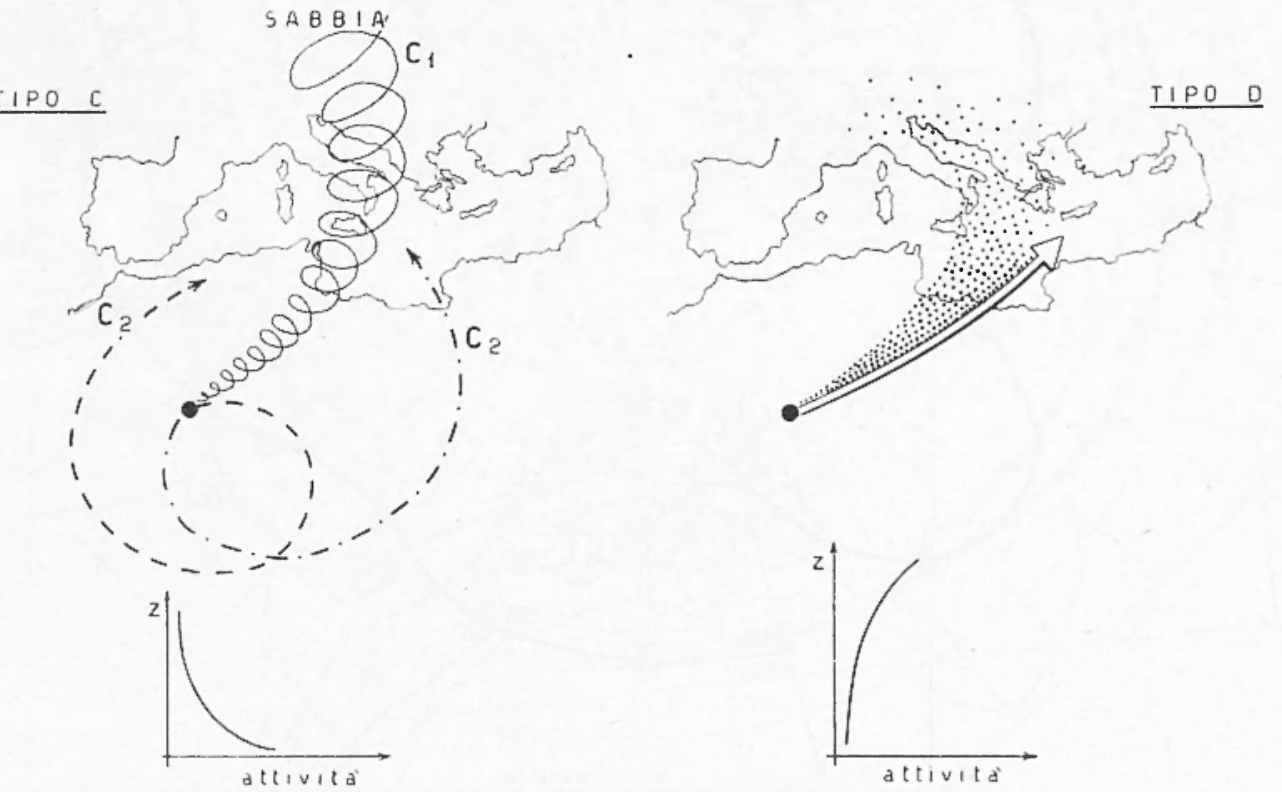

TIPO
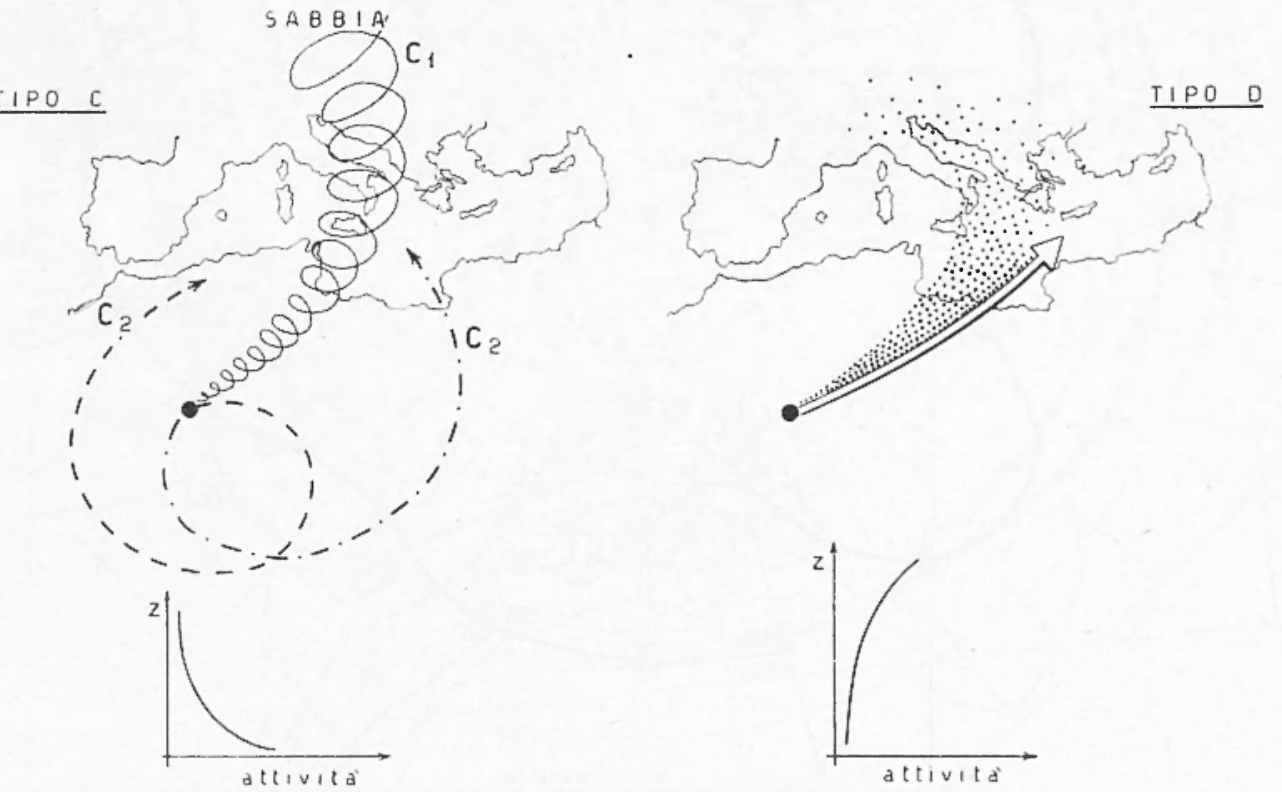

Fig. 1. - Tipi di trasporto di polveri raclioattive 
tivamente lento e l'attività ladioattiva diminuisce rapidamente con la quota.

Tipo C: 'Trasporto sull'Italia di polveri risollevate in condizioni di turbolenza nei bassi strati:

Tipo $\mathrm{C}_{2}$ - Trasporto diretto sull'Italia. In tali casi l'aria affluente contiene notevoli quantità di sabbia di origine sahariana.

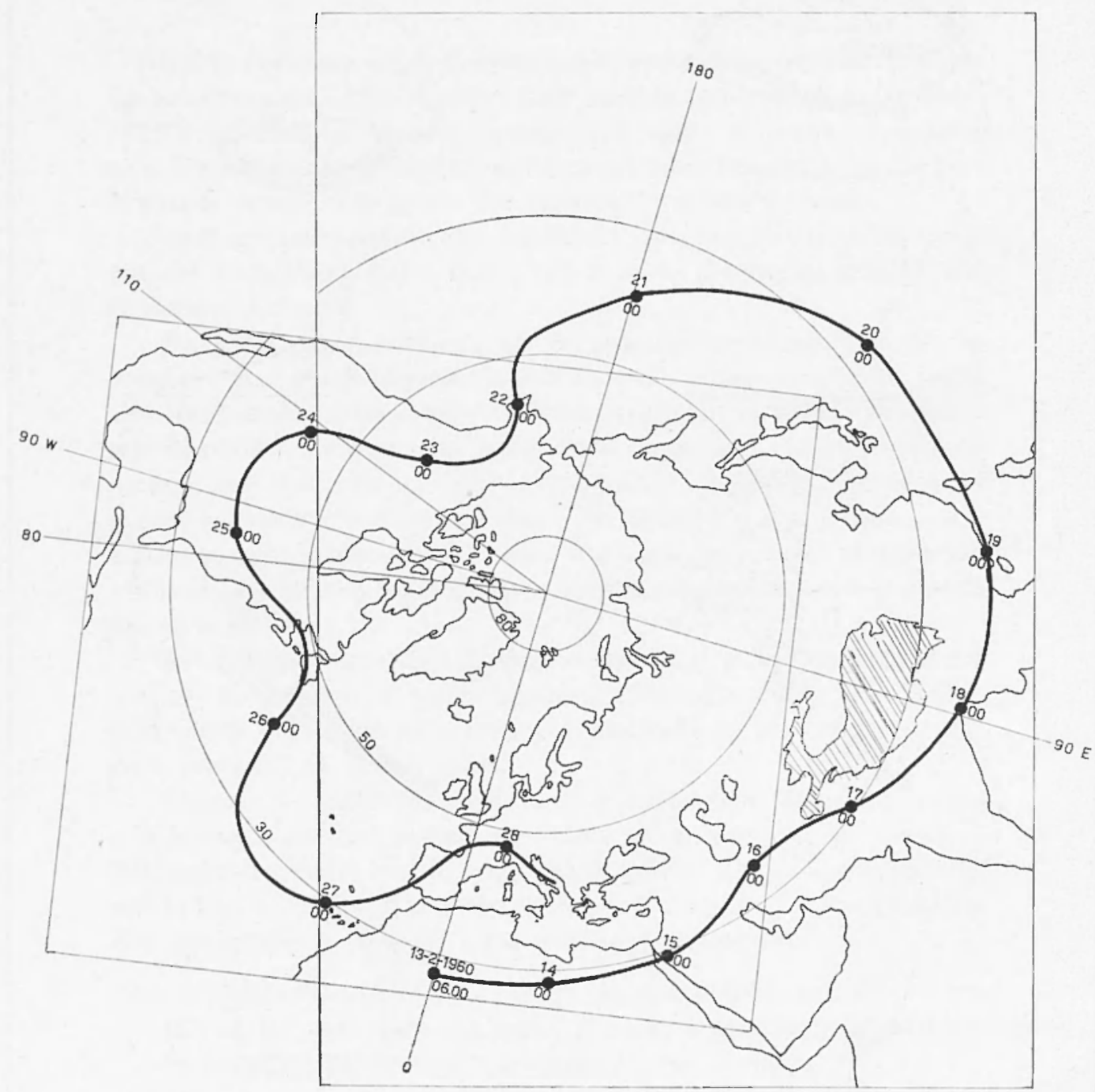

Fig. 2. - Percorso presumibile, a 500 mb, della nube di Tipo A prodotita dalla prima esplosiono sahariana. 
Tipo $C_{1}$ - Trasporto di ritorno sull'Italia, a causa di modeste circolazioni, interessanti i bassi strati, sull'Africa Settentrionale.

Nei casi di trasporto di Tipo $\mathrm{C}$ sono soprattutto interessate le località più vieine all'origine; l'attività radioattiva decresce con lá quota.

Nel lavoro tedesco sono stati presi in considerazione solo trasporti dei tipi da noi classificati come $\Lambda$ e $\mathrm{C}$; in particolare non è stata esami-

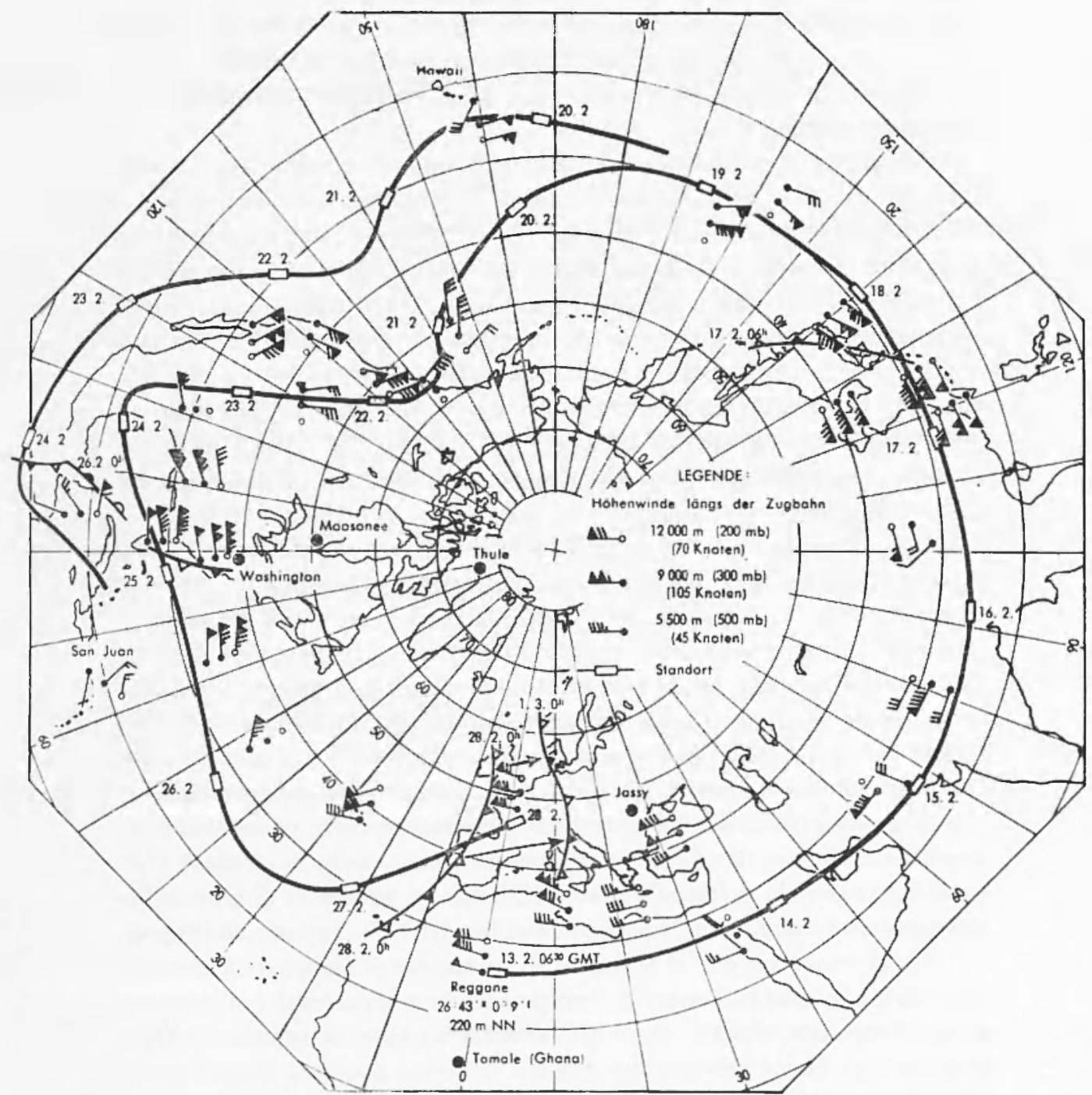

Fig. 3. - Traiettoria dolla nube radioattiva troposferica prodotta dalla prima esplosione sahariana, secondo linterpretazione di Brauer. 
nata la possibilità della formazione di una nube di tipo $\mathrm{B}$, trasportata dagli alisei nei bassi strati della atmosfera. La formazione di nubi di tale tipo era gia stata posta in evidenza in un articolo di P. A. Sheppard ( $\left.{ }^{4}\right)$ sulla dispersione delle particelle radioattive nell'atmosfera. Manca inoltre, nel lavoro di Brauer, ogni considerazione sulla distribuzione verticale della radioattività.

Le traiettorie seguite dalla nube principale di tipo A, conseguente alla prima esplosione atomica, tracciate da noi e dai tedeschi, concordano in modo abbastanza soddisfacente, pur esistendo differenze, dovute a diversità di criteri nel tracciamento ed a inevitabili discordanze di valutazione (Figg. 2 e 3 ).

La prima parte del percorso sino al Giappone è stata tracciata da Brauer sulla base del fatto che il giorno 17 Febbraio venne osservata a Tokio la presenza nelle precipitazioni di attivita beta dovuta a prodotti di fissione recenti; la velocità media con cui la nube avrebbe dovuto viaggiare nell'alta troposfera per raggiungere Tokio a tale data è stata assunta come una misura empirica del trasporto della nube radioattiva; mentre il percorso successivo venne tracciato in base ai venti a $500 \mathrm{mb}$, che furono ritenuti rappresentare in modo soddisfacente l'ordine di grandezza della velocità di trasporto della nube. Noi invece (che non eravamo in possesso dei dati giapponesi) ci siamo limitati a considerare il trusporto nella media troposfera, scegliendo, per tutto il tragitto, il livello di riferimento di $500 \mathrm{mb}$. Il fatto che la nostra traiettoria indichi il passaggio della nube in prossimità del Giappone il giorno 19 , cioè due giorni dopo il 17 , non $\dot{e}$ in contrasto essenziale con le osservazioni, in quanto a Tokio la radioattività fu registrata in corrispondenza del passaggio di un marcato fronte freddo, il quale determinò precipitazioni che trascinarono al suolo i prodotti di fissione esistenti nell'alta troposfera. Ora le correnti a $300 \mathrm{mb}$ erano nettamente più forti che non a $500 \mathrm{mb}$.

Per quanto si riferisce all'arrivo della nube troposferica radioattiva sull'Europa occidentale i dati italiani (che non erano in possesso dei tedeschi) consentono di affermare (riteniamo senza ombra di dubbio) trattarsi del passagroio della nube di tipo $A$, che ha effettuato il giro dell'emisfero e che è giunta sull'Italia con correnti da NW il giorno 28 (Fig. 4).

Líb precisazione che si trattasse di una nube di tipo $A$ si è ritenuta opportuna, in quanto Brauer ha prospettato la possibilità che si trattasse di prodotti direttamente provenienti dalla regione di esplosione, convogliata da un'alta pressione al suolo ed in quota, esistente in quei giorni sull'Africa nord-occidentale; tali prodotti si sarebbero successivamente immessi entro la corrente occidentale principale. 
Tale interpretazione è basata sul presupposto che in quota esistesse ancora sulla regione di esplosione, a tanti giorni da quello dell'esperimento, un'imponente "riserva" di polveri ladioattive. Tale "riserva" si sarebbe formata a causa di noti di subsidenza al disopra dell'inversione, che in genere, nelle regioni subtropicali interessate dalla fascia delle alte pressioni in quota, limita superiormente lo strato convettivo.

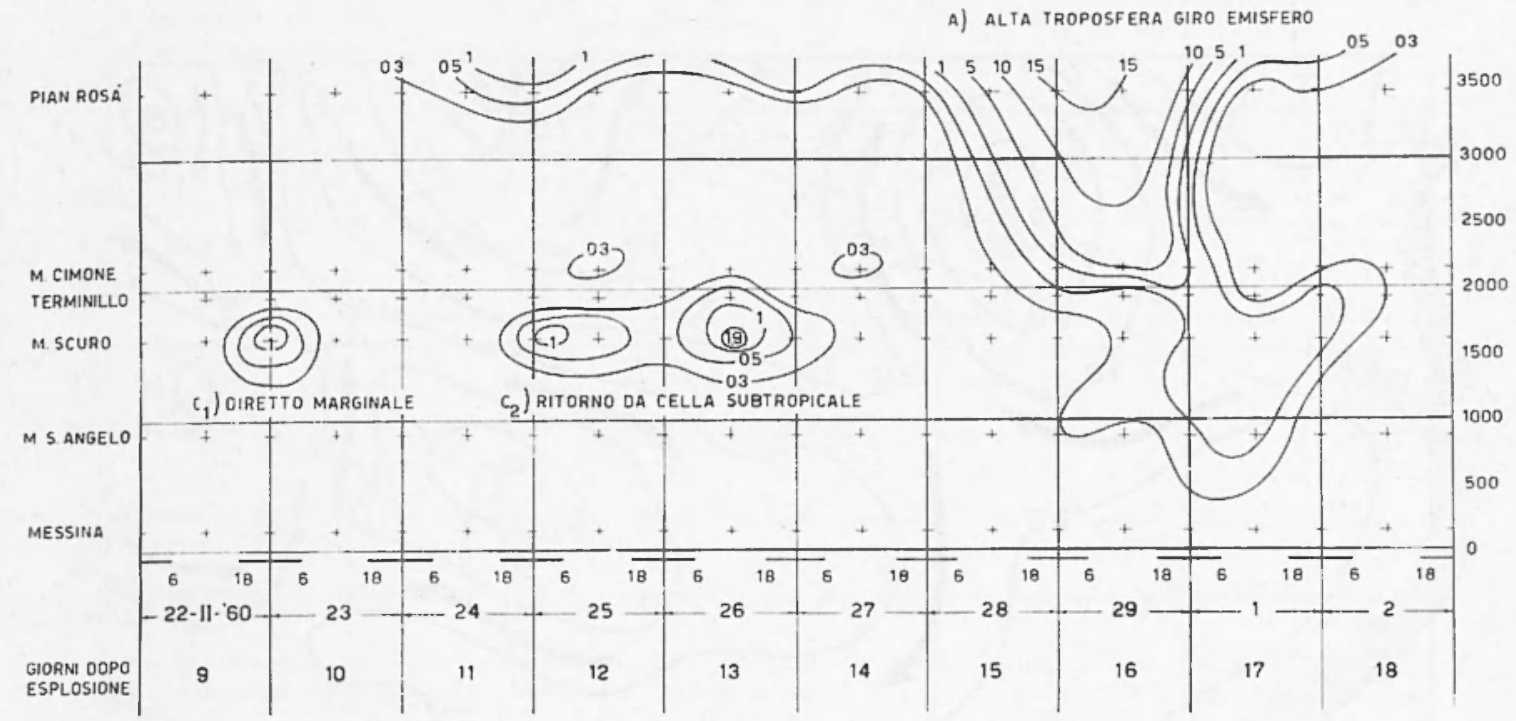

Fìr. 4. - Isoplete di radioattività relative all'Italia (periodo dal 13 Febbraio al 2 Marzo 1960 - valori in $\left.\mathrm{pc} / \mathrm{m}^{3}\right)$.

Tale possibilità, da noi non presa in ronsiderazione, che fu già oggetto di precedenti ricerche $\left(^{5}\right)\left(^{6}\right)$ è altamente suggestiva; essa ci sembra debba essere presa in particolare considerazione nei trasporti di tipo $\mathrm{C}$, nei casi cioc̀ in cui, specialmente in corrispondenza di un passaggio frontale freddo, la distruzione temporanea dell'inversione e la turbolenza potrebbero portare nei bassi strati i prodotti della " riserva " che si agrgiungerebbero a quelli inmessi nell'atmosfera per il risollevamento turbolento di polversi radioattive.

Non ci sembra peraltro giustificabile l'ipotesi che tale "riserva" debba essere stata cosi imponente che i prodotti da essa prelevati, a tanti giorni da quello dell'esplosione. possano determinare valori di radionttività cosi elevati quale quello di $15 \mathrm{pc} / \mathrm{m}^{3}$ registrato a Pian Rosà il giorno 29 Febbraio. 
Is opinioni di Braner e le nostre concordano nell'ammettere che i valori di 0,8 e $0,9 \mathrm{pc} / \mathrm{m}^{3}$ registrati nell'aria a Monaco nei griorni 21 e 22 II (e che l'analisi radiorhimica ha dimostrato essere dovuti a prodotti di fissione recenti) possano attribuirsi a trasporto di tipo $\mathrm{C}$ di polveri sahariane in situazioni di sciroceo e nel ritenere importante l'effetto dei passaggi frontali fredli sul risollevamento di polveri sahariane contaminate.

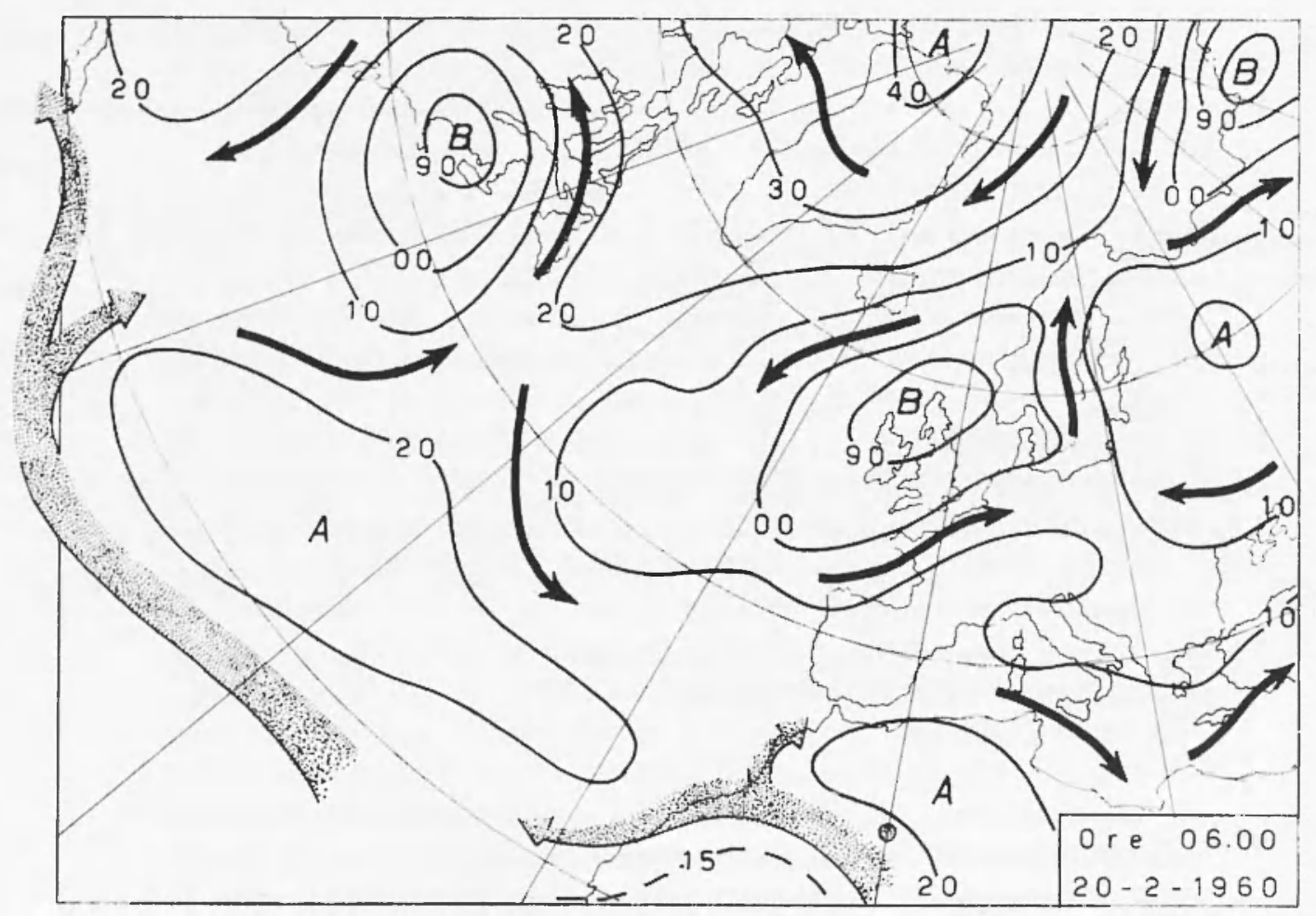

Fig. 5. - Situazione barica al suolo alle ore 00 T.M.G. del 20 Febbraio 1960.

Ina grande perplessità desta invece in noi l'interpretazione texlescal degli alti valori registrati nell'America Centrale. Secondo la nostra interpretazione tali alti valori sono da imputarsi an una nube di tipo $B$, trasportata dagli Alisei nei bassi strati dellatmosfera; per tale nube l'inversione subtropicale di subsidenza agisce escenzialmente come sbapramento per il sollevamento dei prodotti di fissione ai livelli superiori. Si può cosi spiegare la forte concentrazione delle polveri radioattive in 



Fig. 6. - Isoplete di radioattivita a lunga vita secondo i meridiani $110^{\circ} \mathrm{W}$ $\theta 80^{\circ} \mathrm{W}$ (periodo dal 13 Febbraio al 30 Marzo 1960 - valori in $p\left(-\mathrm{mi}^{3}\right.$ ). 
tale nube anche a grande distanza dall'origine. La circolazione predominante nei bassi strati nel periodo considerato può essere bene rappresentata dalla situazione al suolo del 20 Febbraio (Fig. 5). Si osservino in Fig. 6 le isoplete di radioattività dell'aria relative a due sezioni temporali eseguite lungo i meridiani $80^{\circ}$ e $110^{\circ} \mathrm{W}$; in Fig. 7 sono riprodotte le ubicazioni delle località le cui osservazioni sono state utilizzate per l'elaborazione della Fig. 6.

Il fatto che la nube sia pervenuta prima sulla località più orientale (Paramaribo) tra quelle delle latitudini più basse intorno a $20^{\circ}$ Nord e poi alle località più occidentali, (Curaçao prima e Miraflores poi) è una conferma della nostra interpretazione. I bassi valori successivamente

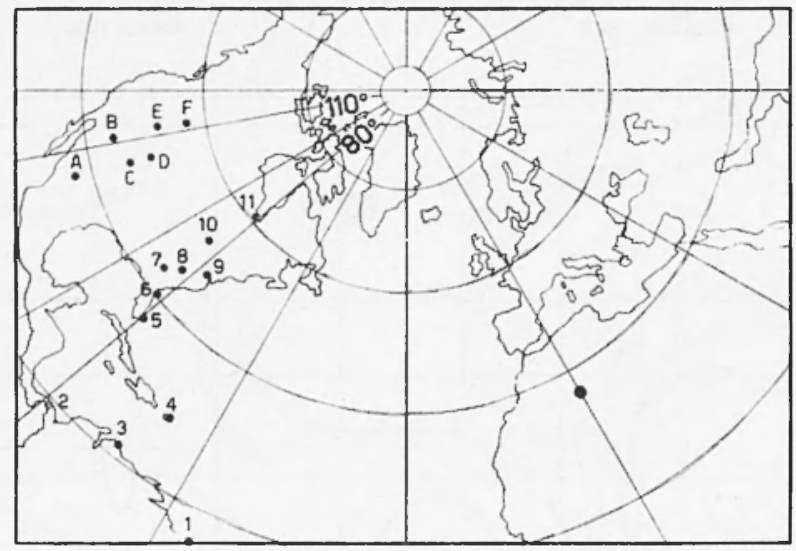

Fig. 7. - Ubicazione delle località i cui dati sono stati utilizzati per traceiare le isoplete di Fig. 6.

registrati a San Juan e Miami sono attribuibili a prodotti di dispersione dalla nube principale di tipo $B$, deviati verso Nord dalle correnti dei bassi strati esistenti sulla regione ai primi di Maızo.

E da notare, a questo riguardo, che non risulta che Brauer fosse at conoscenza dei dati di Curaçao e di Paramaribo (stazioni facenti parte della rete olandese di osservazione) né di quelli degli Stati Uniti forniti dall'U. S. Department of Health, Education and Welfare.

Una conferma a posteriori del nostro punto di vista ci viene offerto dall'articolo stesso di Brauer, che ci fornisce i risultati che Ward e Marr (?) dedussero dalle osservazioni effettuate nel Ghana. A Tamale infatti, $1800 \mathrm{~km}$ a sud di Reggane, giungeva, 3 giorni dopo l'esplosione, una nube radioattiva che faceva registrare l'altissimo valore di 1000 
$\mathrm{pc} / \mathrm{m}^{3}$ e i cui effetti si protraevano per circa 10 giorni. Sono le polveri radioattive contenute in tale nube, che deviate successivamente verso occidente dalle correnti orientali della parte inferiore della cella anticiclonica subtropicale, hanno in seguito raggiunto l'America Centrale

Oltre che per le ragioni precedentemente esposte l'interpretazione di Brauer non ei convince per i seguenti motivi:

a) Una biforcazione del flusso in quota a occidente delle Hawaii può essersi verificata il giorno 19 (rivedasi la Fig. 3); ma ci sembra che il suo effetto non abbia potuto essere altro che quello di produrre una dispersione laterale (sia pure notevole) dalla nube principale di tipo $\mathrm{A}$, dispersione che riteniamo responsabile dei valori registrati a Mauna Loa, nelle Hawaii. Una notevole quantità dei prodotti dispersi avrebbe dovuto però entrare a far parte della circolazione dell'anticiclone subtropicale ed essere risospinta verso l'Asia. La parte dei prodotti trasportata verso levante lungo la nuova traiettoria avrebbe dovuto essere scarsa, molto diffusa, ed avrebbe dovuto spostarsi con velocità nettamente inferiori a quelle relative al percorso più settentrionale.

b) Per quanto i dati meteorologici in nostro possesso relativi all'America Centrale siano scarsi, ei sembra di poter escludere che durante il periodo fine Febbraio-inizio Marzo si siano verificati sia la presenza di una ben definita goccia fredda in quot? (di cui parla l'articolo) sia un imponente e persistente trascinamento al suolo di aria superiore subsidente, estremamente secca. Tale trascinamento avrebbe dovuto avvenire in concomitanza con un nucleo anticiclonico in quota, centrato sulle regioni sud occidentali degli Stati Uniti e sul Messico, alta pressione di cui non esiste traccia a $500 \mathrm{mb}$. Il golfo del Messico è stato invece interessato in tale periodo $\mathrm{d}_{\mathrm{a}}$ zone depressionarie nei bassi strati, associate ab perturbazioni di carattere frontale e nella zona del Mar dei Caraibi hanno predominato, in prossimità del suolo, correnti umide ${ }^{b}$ provenienti da oriente.

\section{RIASSUNTO}

Si espongono alcuni risultati degli studi compiuti presso il Servizio Meteorologico dell'A.M. sui fenomeni troposferici di trasporto e diffusione a grande scala dei prodotti di fissione immessi nell'atmosfera in conseguenza della prima esplosione nucleare Sahariana. 
$S i$ confrontano tali risultati con quelli ricavati da un analogo studio, condotto presso il Servizio Meteorologico Tedesco, discutendo le principali divergenze d'interpretazione.

\section{$S U M M A R Y$}

Some results of studies, carried out at Italian Air Force Weather Service, on tropospheric phenomena of large scale diffusion, and transfer of fission products, merged into the atmosphere as a consequence of first Sahara nuclear blast, are here presented.

These results are then compared with those obtained by a similar study iarried out at German Weather Service, and more important disagreements cn interpretation are finally discussed.

\section{BIBLIOGRAFIA}

(1) Fea G., Gazzola A., Montalto M., Esempi di impiego di traccianti radioattivi nello studio dei moti atmosferici, "Atti del X Convegno dell'Associazione Geofisica Italiana".

$\left({ }^{2}\right)$ - - Ricerche sui moti atmosferici a grande scaln, condotte sulla base di osservazioni della radioativitì a lunga vita, "Rivista di Meteorologia Aeronautica ") 3, (Luglio-Settembre 1961).

(3) Brauer, Von I., Die Terirachtung radioaliver Schwaden nach dem französischen Atomtest in der Sahara vom 13-2-1960, "Atomkernenergio", 1, 6, (1961).

(1) Srieppand, P. A., Dispersione di particelle radioattive nell atmosfera. "Rivista di Meteorologia Aeronautica ", 3, (Luglio-Settembre 1959).

$\left(^{5}\right)$ Neuwrrtir, R., Met. Auswertung von Messungen der liunstlichen Radioaktivität der Luft und des Niederschlajens, "Goofisica pura ed applicata ", 32, (1955).

${ }^{(6)}$ Israel H. a Reifrerscheid H., Bemerkungen zur radioalitiven Terseuchung der Atmosphäre. "Atomenergio", 3, 250, (1958).

(7) Ward and Marr, Radioactive Fallout in Ghana, "Naturo 4739", (July 23, 1960). 